\title{
Mutation Hot Spot Region in the HOGA1 Gene Associated with Primary Hyperoxaluria Type 3 in the Chinese Population
}

\author{
Wenying Wang ${ }^{a} \quad$ Yi Liu $^{b} \quad$ Lulu Kang $^{b} \quad{\text { Ruxuan } \mathrm{He}^{\mathrm{b}}}^{\mathrm{b}}$ Jinqing Song ${ }^{\mathrm{b}}$ \\ Yanhan $\mathrm{Li}^{\mathrm{c}}$ Jun $\mathrm{Li}^{\mathrm{a}}$ Yanling Yang ${ }^{\mathrm{b}}$ \\ aDepartment of Urology, Beijing Friendship Hospital, Capital Medical University, Beijing, \\ China; ${ }^{b}$ Department of Pediatrics, Peking University First Hospital, Beijing, China; \\ 'Department of Laboratory Animal Center, Institute of Hematology and Blood Diseases \\ Hospital, Tianjin, China
}

\section{Keywords}

HOGA1 · HOGA · Kidney stones · Primary hyperoxaluria type 3

\begin{abstract}
Background: Primary hyperoxaluria type $3(\mathrm{PH} 3)$ is a rare autosomal recessive disorder that affects glyoxylate metabolism. PH3 is caused by defects in 4-hydroxy-2-oxoglutarate aldolase, which is encoded by the HOGA1 gene. However, only 3 cases of PH3 have been described in Asians until today. This study aimed to determine the clinical and mutation spectra of patients from mainland China with PH3. Methods: We applied targeted next-generation sequencing to four non-consanguineous, unrelated Chinese families with PH3 to identify the genes hosting disease-causing mutations. This approach was confirmed by Sanger sequencing. Results: Five patients ( 2 boys and 3 girls) from four unrelated Chinese families were admitted because of kidney stones. Five HOGA1 gene sequence mutations were detected, including two novel mutations, c.811C > T (p.R271C) and c.812G >A (p.R271H). These compound heterozygous mutations were detected in a female $\mathrm{PH} 3$ patient (patient 4). Other patients included 2 boys who had heterozygous c.834_834+1GG $>$ TT and c.834G $>$ A (p.A278A) mutations (patients 1 and 2), a girl with homozygous c.834G >A (p.A278A) mutation (patient 3), and a girl with heterozygous c.834_834+1GG>TT and c.346C $>\mathrm{T}$ (p.Q116X) mutations (patient 5). The mutations in the c.834_834+1 region, including c.834G >A, c.834+1G>T, and c.834_834+1GG $>$ TT, account for $5 / 8$ of alleles in our study and $3 / 4$ of alleles reported among Chinese patients. All patients in this study received hyperhydration and urine alkalinization treatment. Conclusion: Five PH3 cases were reported. Potential mutation hot spot region (c.834_834+1) in the Chinese population and two novel mutations were found.
\end{abstract}

This study was presented in an abstract form at the 36th World Congress of Endourology, 2018 (MP2-19), and 38th Congress of the Societé Internationale d'Urologie, 2018 (MP 2-5).

Yanling Yang, MD, PhD

Department of Pediatrics, Peking University First Hospital No.1 Xianmen Street

Xicheng District, Beijing 100034 (China)

E-Mail organic_acid@126.com
Jun Li, MD, PhD

Department of Urology, Beijing Friendship Hospital, Capital

Medical University

No. 95 Yongan Road

Xicheng District, Beijing 100050 (China)

E-Mail lijun_bjfh@126.com 


\section{Kidney \\ Blood Pressure \\ Research}

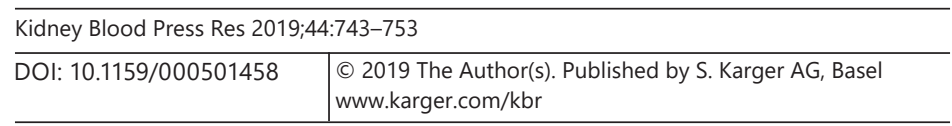

Wang et al.: Mutation Hot Spots Related to PH3

\section{Introduction}

Primary hyperoxaluria type 3 (PH3; MIM 613616), a rare autosomal recessive disorder that affects glyoxylate metabolism, is caused by defects in the HOGA1 gene (OMIM 613597), located on 10q24.2 [1]. Based on the genomic data, specifically a mutant allele frequency study, the prevalence of PH3 is currently estimated to be 1:136,000 [2]. Also, PH3 is believed to be as prevalent as primary hyperoxaluria type 1 (PH1). Exome sequencing (Exac database) has detected many additional rare and potentially damaging mutations that had not been reported previously, potentially indicating increasing prevalence [3]. However, there are few PH3 cases reported worldwide, and only 3 affected individuals have been reported in the Chinese population, which suggests that PH3 cases are underdiagnosed and/or incompletely penetrant $[3,4]$.

The HOGA1 gene encodes a liver-specific mitochondrial enzyme, 4-hydroxy-2-oxoglutarate aldolase (HOGA; $35 \mathrm{kDa}, 327$ amino acids), which catalyzes the transamination of 4-hydroxy-2-oxoglutarate (HOG) to glyoxylate and pyruvate [1]. A deficiency in this enzyme is responsible for HOG accumulation in the mitochondria. The excess HOG is transported to the cytosol and later converted into oxalate. Also, the inhibition of glyoxylate reductase leads to oxalate accumulation due to accumulating HOG $[1,5]$. About only $10 \%$ of urinary oxalate is derived from dietary oxalate, while the remaining $90 \%$ is endogenous oxalate, which is produced via the metabolism of oxalate precursors [6]. In primary hyperoxaluria ( $\mathrm{PH})$, the elevated level of endogenous oxalate, which forms insoluble calcium oxalate crystals, consequently contributes to oxalate precipitation throughout the body, particularly in the kidneys, bladder, eyes, heart, bones, and sometimes the lungs, which leads to cell damage and organ dysfunction. Thus, like other PH types, the typical presentation of PH3 is recurrent urolithiasis. However, systematic oxalosis is absent in $\mathrm{PH} 3$ patients [7], and hypercalciuria serves as a marked phenotype of $\mathrm{PH} 3$ that is rare in $\mathrm{PH} 1$ and $\mathrm{PH} 2$. $\mathrm{PH} 3$ is the mildest type of $\mathrm{PH}$, with good preservation of kidney function.

At the time of this writing, only 3 cases of $\mathrm{PH} 3$ have been reported in the Chinese population $[4,8]$, and no patients have been reported in other populations in Asia. The related articles were found on the PubMed website (https://www.ncbi.nlm.nih.gov/pubmed/) by searching the following keywords: primary hyperoxaluria type 3, China, Chinese, and HOGA1. The PH3 mutation spectrum in China has not yet been established, and no mutation hot spots have been detected.

Here, we report the clinical, biochemical, and potentially causative mutations in 5 Chinese PH3 patients, including potential hot spot mutation region (c.834_834+1) and two novel mutations, thereby expanding the mutation spectrum for this disease. We are now performing genetic and biochemical tests on additional patients who developed kidney stones early in their lives.

\section{Materials and Methods}

\section{Patients}

Five patients ( 2 boys and 3 girls, age range: 1 year and 9 months to 5 years and 2 months) from four unrelated Chinese families were diagnosed at the Department of Pediatrics, Peking University First Hospital. The clinical onset of symptoms occurred between 6 months and 1 year of age. The patients were admitted with indications of hematuria, frequent urination, slow urination, and dysuria. One of our patients (patient 2) came to the hospital because of positive family history. The parents of all patients were healthy and non-consanguineous. 
Table 1. Clinical and laboratory data of five Chinese patients with PH3

\begin{tabular}{|c|c|c|c|c|c|c|}
\hline & \multicolumn{5}{|l|}{ Patient } & \multirow[t]{2}{*}{ Normal range } \\
\hline & 1 & 2 & 3 & 4 & 5 & \\
\hline Sex & $\mathrm{M}$ & M & $\mathrm{F}$ & $\mathrm{F}$ & $\mathrm{F}$ & \\
\hline Age at onset & $6 \mathrm{~m}$ & $3 y$ & $10 \mathrm{~m}$ & $5 y$ & $9 \mathrm{~m}$ & \\
\hline Age at diagnosis & $7 \mathrm{~m}$ & $3 y$ & $4 y$ & $5 y$ & 1 y 3 m & \\
\hline Present age & $10 \mathrm{~m}$ & 3 y 2 m & 4 y $5 \mathrm{~m}$ & 5 y $2 \mathrm{~m}$ & 1 y $9 \mathrm{~m}$ & \\
\hline Positive family history & - & & - & - & - & \\
\hline \multicolumn{7}{|l|}{ Symptoms and signs } \\
\hline Hydronephrosis & + & - & + & + & - & \\
\hline Hypercalciuria & $\mathrm{N} / \mathrm{A}$ & $\mathrm{N} / \mathrm{A}$ & + & $\mathrm{N} / \mathrm{A}$ & + & \\
\hline Proteinuria & - & - & - & + & + & \\
\hline Kidney stones & + & - & + & + & + & \\
\hline Multiple urolithiasis & - & + & - & + & - & \\
\hline Repeated urinary infection & - & - & - & + & - & \\
\hline Chronic renal failure & - & - & - & - & - & \\
\hline \multicolumn{7}{|l|}{ Laboratory findings } \\
\hline Serum creatinine, $\mu \mathrm{mol} / \mathrm{L}$ & 36.4 & 48.2 & 41.2 & 41.5 & 39.8 & $24.9 \sim 69.7$ \\
\hline Urine oxalate, $\mathrm{mg} / 1.73 \mathrm{~m}^{2} \times 24 \mathrm{~h}$ & $\mathrm{~N} / \mathrm{A}$ & $\mathrm{N} / \mathrm{A}$ & $227.49 \uparrow$ & $160.15 \uparrow$ & $197.62 \uparrow$ & $<45$ \\
\hline Outcome & healthy & healthy & healthy & healthy & healthy & \\
\hline
\end{tabular}

\section{Routine Tests and Metabolic Studies}

Routine blood and urine laboratory tests were conducted, including electrolytes, liver, and renal function; glucose; ammonia; ketones; thyroid hormone; creatine kinase and creatine kinase isoenzymes. Transabdominal ultrasonography, urinary system ultrasonography, and abdominal computed tomography were performed.

Urinary oxalate was analyzed via gas chromatography-mass spectrometry, which was performed using a GCMS-QP2010 analyzer (Shimadzu, Japan).

\section{HOGA1 Analysis}

We obtained informed consent from the parents of pediatric patients before genetic analysis. Peripheral blood samples were collected from all patients and their parents, and lymphocytes' genomic DNA was extracted using a TIANamp blood DNA kit (Tiangen Biotech, China). We performed exome sequencing (Agilent V5, US) for genes related to PH3. The exons and flanking intronic regions of the mutations detected in HOGA1 were amplified via polymerase chain reaction and then sequenced. The sequence data were compared with the reference sequence of HOGA1 (NM_138413) to detect the mutations. Mutations were compared to HGMD datasets to identify previously reported mutations (http://www.hgmd.cf.ac.uk). Then, the mutations were compared to the 1000 Genomes Project (http://www.1000genomes.org) and ExAC browser (http://exac.broadinstitute. org) to determine the minor allele frequency (MAF) and then rule out those mutations with high MAF values.

Prediction of the Effects of HOGA1 Sequence Mutations and Conservation Analysis

Multiple sequence alignments were analyzed to determine the degree of conservation of the novel mutations. PolyPhen-2 (http://genetics.bwh.harvard.edu/pph) and MutationTaster (http://www.mutationtaster.org) were used to predict the impact of mutations on protein function. 


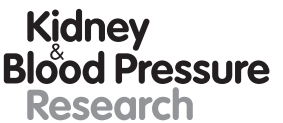

Research

\begin{tabular}{|c|c|}
\hline \multicolumn{2}{|c|}{ Kidney Blood Press Res 2019;44:743-753 } \\
\hline DOI: $10.1159 / 000501458$ & $\begin{array}{l}\text { (c) } 2019 \text { The Author(s). Published by S. Karger AG, Basel } \\
\text { www.karger.com/kbr }\end{array}$ \\
\hline
\end{tabular}

Wang et al.: Mutation Hot Spots Related to PH3

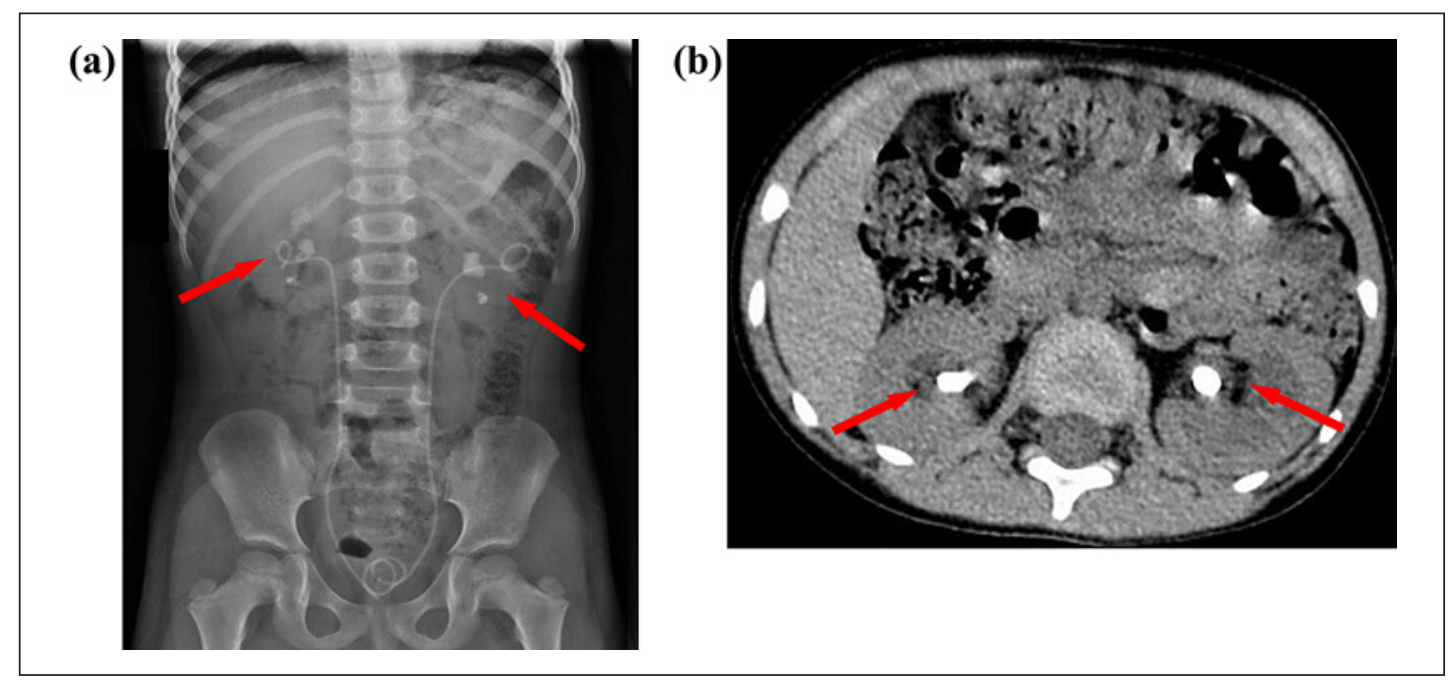

Fig. 1. Imaging studies in patient 4 who is harboring two novel HOGA1 mutations. Abdominal X-ray (a) and low-dose contrast-enhanced CT scan (b) showing bilateral stones (arrows).

\section{Results}

The clinical data and the results of the laboratory examinations are presented in Table 1. All patients were born at term from healthy unrelated parents. Patients 1 and 2 were siblings; patient 1 is a boy aged 1 year and 3 months born via normal delivery. The boy presented with dysuria at the age of 6 months. Ultrasonography of the urinary system revealed bilateral kidney stones, ureterolithiasis, and hydronephrosis. Percutaneous nephrostolithotomy (PCNL) was performed. A genetic diagnosis was obtained when this patient was 10 months old. After diagnosis, he was given a high level of fluid intake (1.5-3 L/day), hydrochlorothiazide, and oral potassium citrate according to treatment guidelines, avoiding excessive vitamin $\mathrm{C}$ and oxalate-rich food intake. Since treatment, his stones have not been aggravated, and the patient's estimated glomerular filtration rate (eGFR) has remained relatively stable. Patient 2 came to our hospital for a health examination at the age of 3 years, after patient 1 diagnosis. Cystic calculus was detected, and urine oxalate and urine citrate acid levels similar to those observed in patient 1 were found. Transurethral cystolithotripsy was performed. Since hydration and urine alkalinization treatment, the patient's stones have not recurred, and his eGFR has remained stable. However, the follow-up duration is not long enough; more time is required to evaluate the effects of treatment.

Patient 3 is a girl aged 4 years and 5 months born via normal delivery who first showed PH3 symptoms (recurrent hematuria and dysuria) at age 3. She suffered from frequent urination when she was 4 years old. Hematuria, slow urination, and dysuria developed later. Bilateral kidney stones and elevated urine oxalate were found, and extracorporeal shock wave lithotripsy (ESWL) was performed when this patient was 4 years old. Her family came to our hospital after the surgery. Elevated urine $\mathrm{Ca}^{2+}$ and uric acid levels and hydronephrosis were detected, and HOGA1 gene mutations were identified. She was diagnosed with a kidney stone, urolithiasis, hydronephrosis, hyperuricemia, hypercalciuria, and PH3. Her prescriptions were the same as those for patient 1 . After 5 months of treatment, her stones and kidney function remained stable.

Patient 4 is a 6-year-old girl born via natural delivery. She first presented with hematuria at the age of 5 years. Kidney stones (Fig. 1), increased urine oxalate, and decreased urine citric 
(a) Patient 1 and 2

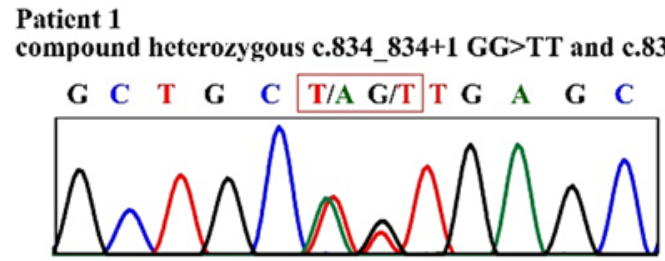

Patient 2

compound heterozygous $c .834 \_834+1$ GG $>$ TT and $c .834 G>A$

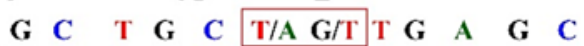

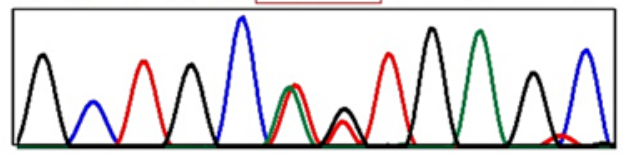

(b) Patient 3

homozygous c.834G $>A$

\begin{tabular}{lllllllllllll} 
G & C & T & G & C & A & G & T & G & A & G & C \\
\hline
\end{tabular}

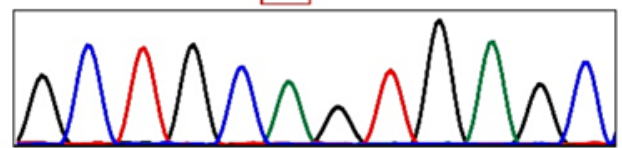

(d) Patient 5

heterozygous c.346C $>T$

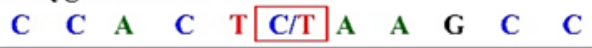

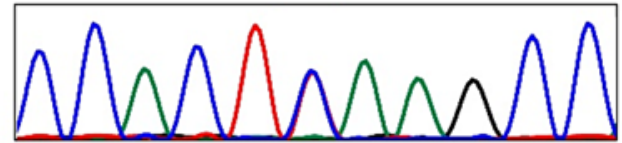

Mother of the proband

heterozygous c.834 G>A

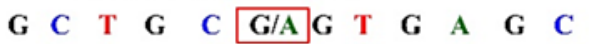

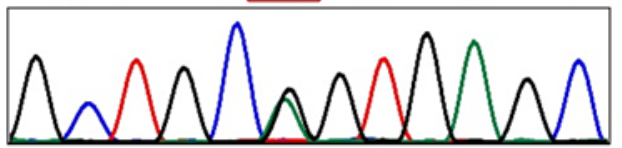

Father of the proband

heterozygous c.834_834+1 GG $>$ TT

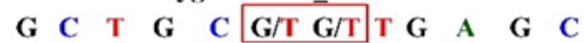

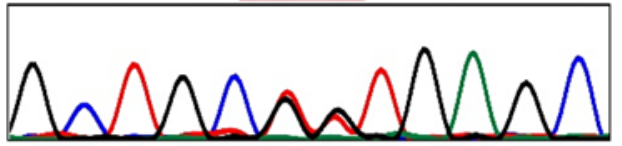

(c) Patient 4

compound heterozygous $\mathrm{c} .811 \mathrm{C}>\mathrm{T}$ and $\mathrm{c} .812 \mathrm{G}>\mathrm{A}$

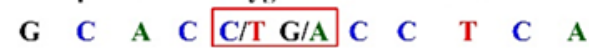

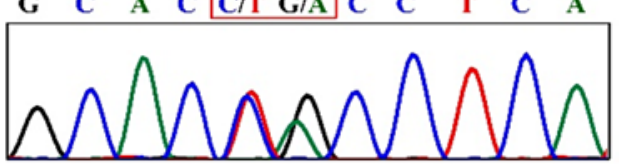

heterozygous c.834 834+1 GG>TT

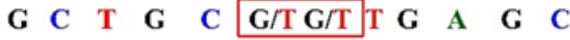

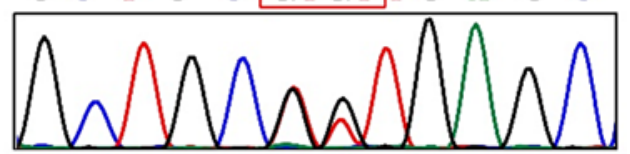

Fig. 2. HOGA1 mutation analyses of our 5 PH3 patients and parents of patients 1 and 2. a Compound heterozygous c.834_834+1 GG>TT and c.834G>A (p.A278A) mutations in patients 1 and 2 and their parents. b Homozygous c.834G>A (p.A278A) mutation in patient 3. c Compound heterozygous c.811C>T (p.R271C) and c.812G $>$ A (p.R271H) mutations in patient 4. d Compound heterozygous c.346C > T and c.834_834+1 GG $>$ TT mutations in patient 4 ; all the parents of our patients are carriers.

acid levels were detected; she was quickly treated via mini-PCNL twice. Her family came to our hospital after the surgery, and she then received her genetic diagnosis. She was diagnosed with a kidney stone, hydronephrosis, and PH3. After 3 months of similar treatment as compared to patient 1 , the girl's stones were not aggravated, and her kidney function remained stable.

Patient 5 is a girl who was born via cesarean because pelvic inlet stenosis was found in her mother. She is 5 years and 8 months old as of this writing. Patient 5 presented to our hospital with a chief complaint of dysuria and hematuria when she was 4 years old. Laboratory tests showed elevated blood white blood cells, increased urine oxalate and $\mathrm{Ca}^{2+}$ in the blood, and normal urea nitrogen, creatinine, and uric acid. After whole-exome sequencing, the patient was diagnosed with kidney stones, urinary tract infection, and PH3. Anti-infective treatment and, subsequently, ESWL were performed. After 8 months of treatment, her condition was stable.

Five mutations were identified across all 4 patients (Table 2; Fig. 2). Compound heterozygous c.834_834+1GG>TT and c.834G>A (p.A278A) mutations in patients 1 and 2 (Fig. 2a), a homozygous c.834G $>$ A (p.A278A) mutation in patient 3 (Fig. 2b), compound heterozygous 
Kidney

Blood Pressure

Research
Kidney Blood Press Res 2019;44:743-753 DOI: 10.1159/000501458

(c) 2019 The Author(s). Published by S. Karger AG, Basel www.karger.com/kbr

Wang et al.: Mutation Hot Spots Related to PH3

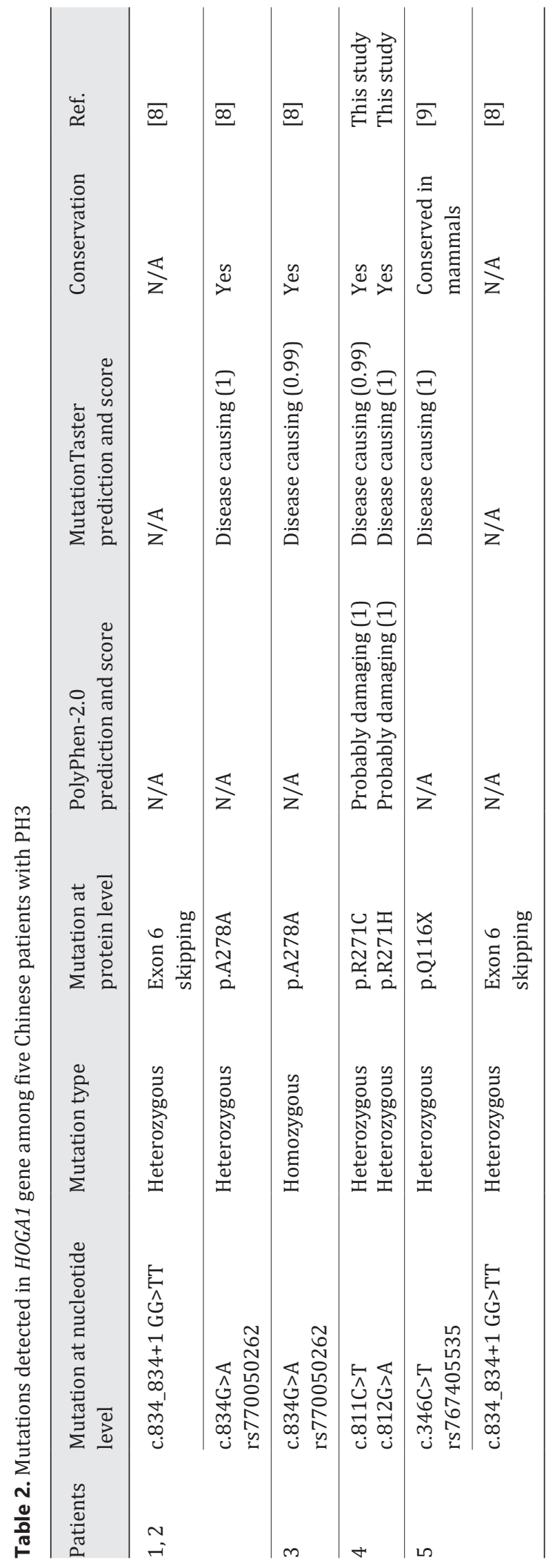




\section{Kidney \\ Blood Pressure \\ Research}

Table 3. Amino acid alignment in HOGA residues (highlighted in bold)

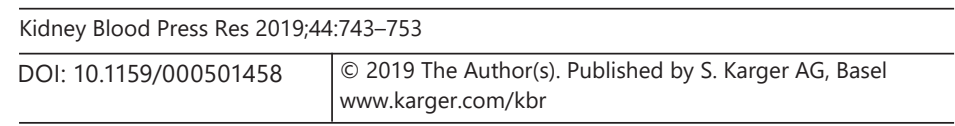

\begin{tabular}{ll}
\hline Species & $\begin{array}{l}\text { HOGA sequences } \\
\text { (the 271st amino acid of HOGA) }\end{array}$ \\
\hline Humans & AQKLQHRLIEPNA \\
Macaca mulatta & AQKLQHRLIEPNA \\
Felis catus & AQKLQHRLIEPNA \\
Mus musculus & AQELQHRLIEPNT \\
Trubripes & ARALQQRLIEPNA \\
Danio rerio & --- - YRLIEPNT \\
Xenopus tropicalis & AKELQYRLIEPNT \\
\hline
\end{tabular}

c.811C > T (p.R271C) and c.812G >A (p.R271H) mutations in patient 4 (Fig. 2c), and compound heterozygous c.346C $>$ T (p.Q116X) and c.834_834+1GG>TT mutations in patient 4 (Fig. 2d) were found. All the mutations were detected in each parent of patients. Among these mutations, only two mutations, c.811C > T (p.R271C) and c.812G $>$ A (p.R271H), were novel. The otherthreemutations, c.346C>T(p.Q116X)[9],c.834G >A(p.A278A)[8], andc.834_834+1GG>TT [8], had been previously reported.

The novel mutations [c.811C>T (p.R271C) and c.812G>A (p.R271H)] and c.834_834+1GG>TT are found neither in 1000 Genomes Project nor in the ExAC program, while the other two mutations [c.346C $>$ T (p.Q116X) and c.834G $>$ A (p.A278A)] are pathogenic polymorphisms (Table 2). The PH3 sequence alignment revealed that amino acid residue R271 is conserved among humans, Macaca mulatta, Felis catus, Mus musculus, Takifugu rubripes, Danio rerio, Drosophila melanogaster, and Xenopus tropicalis (Table 3). Two missense mutations (p.R271C and p.R271H) are predicted to be "probably damaging" and "diseasecausing," respectively, by PolyPhen-2.0 and MutationTaster. The stop gain mutation ( $\mathrm{p}$. Q116X) is predicted to be "disease-causing" by MutationTaster (Table 2), while the c.834_834+1GG>TT and p.A278A mutations cannot be labeled by software because they stretch over exons and introns.

\section{Discussion}

To date, only 3 cases of PH3 have been reported in the Chinese population [4, 8], and no mutation hot spots were identified in the Chinese population, although hotspot mutations have been found in other populations. In this study, we presented 5 cases of PH3 in Chinese patients and described their symptoms, clinical findings, treatments, and responses. The findings will be highly useful in treating future cases of PH3 in the Chinese population. Furthermore, we found the potential mutation hot spot region (c.834_834+1) and two novel mutations [c.811C>T (p.R271C) and c.812G>A (p.R271H)] associated with PH3.

PH3 was first recognized in 2010 by Belostotsky et al. [1]. The clinical features of PH3 include urolithiasis, hypercalciuria, and hyperuricemia [10, 11]. In PH, stones are caused by increased production and accumulation of oxalate and subsequent hyperoxaluria deposition. In addition to stones, systemic calcium oxalate crystal deposition also leads to vascular disease, cardiomyopathy, and retinal disease. However, unlike in other types of PH, systemic oxalosis has not been reported to date in PH3 [12], even in elderly individuals [13].

Frequent and active stones can damage kidney function. However, kidney function is better preserved among PH3 patients as compared to other forms of PH [9]. Only a few cases of chronic kidney disease were reported [4], and end-stage renal disease (ESRD) is even rarer [2]. However, ESRD was found in both children and adult individuals, specifically an 8-year-old 


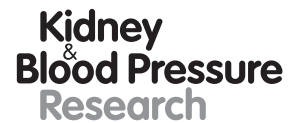

\begin{tabular}{l|l}
\hline Kidney Blood Press Res 2019;44:743-753 \\
\hline DOI: 10.1159/000501458 & $\begin{array}{l}\text { (c) 2019 The Author(s). Published by S. Karger AG, Basel } \\
\text { www.karger.com/kbr }\end{array}$ \\
\hline
\end{tabular}

Wang et al.: Mutation Hot Spots Related to PH3

boy [2] and a 78-year-old man who developed recurrent urolithiasis for some 30 years [13]. Despite oxalate deposition, stone removal procedures and urinary tract obstruction may contribute to kidney function damage. To date, nephrocalcinosis has been reported only occasionally in individuals with PH3 $[11,14]$. PH3 may occur at various ages. $50-65 \%$ of affected individuals develop their first symptoms, such as significant stone manifestations, before the age of 5 years, and stone formation can occur throughout their lives. However, the frequency and severity of stone activity seem to be relieved during adolescence and adulthood $[9,15]$. Thus, some scientist and doctors cautiously considered PH3 to be a benign condition because of the limited number of PH3 cases with long-term follow-ups [9].

In our study, all of our 5 patients had early onset in infancy or early childhood. Four of them had typical kidney stones, with normal kidney function. One patient (patient 2) did not present with a typical phenotype but was identified because of positive family history. Two of the 5 patients (patients 3 and 5 ) had hypercalciuria. Urinary $\mathrm{Ca}^{2+}$ was not estimated in the other 3 patients. Also, repeated urinary infection, proteinuria, and hydronephrosis were observed, which may have resulted from stones, damage, and blockage. Elevated urine oxalate was observed in 3 of the 5 patients (patients 3-5), while patient 1 and 2 did not receive the test. The symptoms are identical with PH3, which makes a differential diagnosis with other inherited kidney stones, and treatment more accessible.

PH3 is initially diagnosed based on clinical features, biomarkers, liver enzyme analysis, and genetic testing. Elevated urinary and plasma oxalate excretion contributes to its biochemical diagnosis. An elevated rate of urinary oxalate accumulation $(>0.7 \mathrm{mmol} / 1.73$ $\mathrm{m}^{2} /$ day) is the most typical biochemical marker, and increased plasma oxalate concentrations ( $>20 \mu \mathrm{mol} / \mathrm{L})$ are very suggestive of PH3 in patients with reduced kidney function [7]. Among the PHs, about $70 \%$ of cases are $\mathrm{PH} 1,10 \%$ are $\mathrm{PH} 2,10 \%$ are $\mathrm{PH} 3$, and others remain unclear in terms of genetic basis [2]. All three types of PH involve high levels of plasma and urine oxalate, though PH3 always involves a lower oxalate accumulation level. Plasma and urine oxalate levels cannot serve as markers of differential diagnosis, but other biochemical tests for the metabolite biomarkers of PH3 make differential diagnosis possible. Urine HOG can be measured via ion chromatography-mass spectrometry [5]; also, HOG and 2,4-dihydroxyglutaric acid can be tested via gas chromatography-mass spectrometry [16]. The 4-hydroxyglutarate test was developed using flow injection tandem mass spectrometry [3]. Urine glycolate estimation helps to identify PH1, and L-glycerate is involved in detecting PH2. Also, glycolate is sometimes detected in $\mathrm{PH} 3$ patients. Furthermore, regarding stone component, stones formed by PH3 patients contain aggregated calcium-oxalate dihydrate crystals that range widely in size [17].

To date, only 33 mutations have been reported in HOGA1 (http://www.hgmd.cf.ac.uk/ ac/index.php). Common HOGA1 mutations, including a deletion (c.944_946 del AGG, p. E315del) and a splice site mutation (c.700+5 G>T), accounted for $74.2 \%$ of total mutations [2, 10]. However, these mutations were not found in our study. A total of 8 patients ( 3 in the previous study, and 5 in our study) were detected in the Chinese population [4, 8]; mutation c.834 G>T accounts for four (one in the previous study [8] and three in this study) of twelve alleles (the siblings in our study and the previous study [8] separately share the same alleles). Also, c.834_834+1GG>TT accounts for three (one in a previous study [8] and two in this study) of twelve alleles, while c.834+1 G>T accounts for one (one in a previous study [4]) of twelve alleles. In total, mutations in c.834_834+1 region account for 8 of 12 alleles among Chinese PH3 patients.

The c.834G>A (p.A278A) mutation, which was detected in 3 of the 5 patients in this study (patients 1-3) creates a synonymous change. This mutation has been reported in Chinese patients before [8], and it creates two types of transcripts. The majority transcript eliminates exon 6 , while the minority transcript retains it as normal. Thus, the codon change results in a 


\section{Kidney \\ Blood Pressure \\ Research}

\begin{tabular}{l|l}
\hline Kidney Blood Press Res 2019;44:743-753 \\
\hline DOI: 10.1159/000501458 & $\begin{array}{l}\text { ○ 2019 The Author(s). Published by S. Karger AG, Basel } \\
\text { www.karger.com/kbr }\end{array}$ \\
\hline
\end{tabular}

Wang et al.: Mutation Hot Spots Related to PH3

decreased splice site recognition ability in c.834, and this position is an important controller of HOGA1 gene splicing [8]. The next mutation, c.834_834+1GG>TT, was also identified from patients 1,2 , and 5 . This complex mutation is a successive two-nucleotide substitution at the last position of exon 6 (the same position as p.A278A) and the first position of intron 6 . This mutation causes the complete skipping of exon 6 [8]. The mutations of patients 1 and 2 are complex; the pedigree analysis makes the mutation identification easier (Fig. 2a). These siblings have the same mutation as a boy who firstly presented with nephrolithiasis at 33 months of age. The boy's condition was very severe, and bilateral renal calculi, multiple bladder stones, and upper ureteral calculi were detected. He later received PCNL of the right kidney, combined with bladder lithotripsy, and of the left kidney. In contrast to the reported case, our siblings (patients 1 and 2 ) represented milder phenotypes, especially patient 2 , who did not show typical symptoms before the health examination. Furthermore, c.834+1 G>T was reported in both Chinese [4] and Caucasian populations [18]. One of the boys who harbored heterozygous c.834+1 G>T and p.C257G mutations showed stage 2 chronic kidney disease (CKD 2) after 10 years of treatment, while his sibling with the same mutation presented with normal eGFR over 8 years of follow-up [4].

The novel mutations, p.R271C and p.R271H, are different changes in the same amino acid. They are predicted to be pathogenic by Polyphen-2 and MutationTaster, and R271 is conserved among species, which indicates that these mutations are likely pathogenic. Homozygous c.346C $>\mathrm{T}$ was identified from a Turkish boy who initially presented with recurrent urolithiasis at 1 year of age. He underwent PNCL and ESWL for curation, and the disease was completely cured after 10 years of follow-up [9]. To date, the genotype-phenotype correlation has not been established, even for the common mutation c.944_946 del AGG and c.700+5 G>T, which may be due to the small patient populations and other genetic/environmental modifier effects [1]. Some pathogenic mutation carriers are idiopathic stone formers, suggesting sensitivity to haploinsufficiency [2,12]. In our study, although a mutation hotspot in this population was identified, the genotype-phenotype correlations remain remote, which might necessitate long-term follow-ups and patient population enlargement.

PH3 patients require lifelong treatment to retard calcium oxalate precipitation, including hyperhydration and urine alkalinization. Hyperhydration helps reduce urine supersaturation with calcium oxalate. A high oral fluid intake $\left(>2.5 \mathrm{~L} / \mathrm{m}^{2}\right.$ body surface area) is recommended [7]; urine alkalinization, via potassium or sodium citrate, orthophosphate, or magnesium, can inhibit calcium oxalate crystallization. Other recommendations in terms of reducing calcium oxalate deposition include a low-salt and high-fiber diet and relatively low vitamin $\mathrm{C}$ intake because excessive quantities of vitamin $\mathrm{C}$ may result in the precipitation of calcium oxalate. Also, marked dietary oxalate intake should be avoided [7]. The treatment of hypercalciuria, including thiazides or a combination of thiazides with amiloride, can significantly reduce urinary supersaturation with calcium oxalate [12].

Symptomatic treatment and daily prevention are also needed. Firstly, urinary tract obstruction should be treated via stent placement and stone removal in time to avoid complications such as infection. Secondly, urinary tract infections should be promptly and thoroughly treated because bacteria may cause pyelonephritis and recurrent infections. Also, acute kidney injury should be avoided by preventing intravascular volume contraction and nephrotoxic agent intake [7]. Here, patients underwent hyperhydration, urine alkalinization, and urinary calcium-reducing treatment. All of them were advised to avoid excessive vitamin $\mathrm{C}$ and oxalate intake. The stones were not aggravated, and all patients showed normal eGFRs. However, because the follow-up duration was not long enough, we require more time to estimate treatment response.

In conclusion, this study reports a potential mutation hot spot region (c.834_834+1) in the Chinese population, and two novel mutations were identified (p.R271C and p.R271H). 
These results will provide a framework for future genetic diagnoses of this disease. To investigate the mutation spectra of disease-causing genes associated with $\mathrm{PH} 3$ in the Chinese population, a large-scale study of PH3 in China should be performed.

\section{Acknowledgments}

We would like to thank all patients who participated in this study for their cooperation.

\section{Statement of Ethics}

This study was approved by the institutional review board of the Department of Pediatrics, Peking University First Hospital, and it was conducted according to the Declaration of Helsinki.

\section{Disclosure Statement}

The authors have no conflicts of interest to declare.

\section{Funding Sources}

This work was supported by the grants from Beijing Municipal Science and Technology Commission (Z181100001718173), Beijing Municipal Administration of Hospitals Clinical Medicine Development of Special Funding Support (XMLX201826), The Capital Health Research and Development of Special Funding (2018-2-1101), and the 13th Five-year Plan National Key Technology R\&D Program from the Ministry of Science and Technology (Grant No. 2017YFC1001700).

\section{References}

1 Belostotsky R, Pitt JJ, Frishberg Y. Primary hyperoxaluria type III - a model for studying perturbations in glyoxylatemetabolism. J Mol Med (Berl). 2012;90(12):1497-504.

2 Hopp K, Cogal AG, Bergstralh EJ, Seide BM, Olson JB, Meek AM, et al. Phenotype-Genotype Correlations and Estimated Carrier Frequencies of Primary Hyperoxaluria. J Am Soc Nephrol. 2015;26(10):2559-70.

3 Greed L, Willis F, Johnstone L, Teo S, Belostotsky R, Frishberg Y, et al. Metabolite diagnosis of primary hyperoxaluria type 3. Pediatr Nephrol. 2018 2018-08-01;33(8):1443-6.

4 Allard L, Cochat P, Leclerc AL, Cachat F, Fichtner C, De Souza VC, et al. Renal function can be impaired in children with primary hyperoxaluria type 3. Pediatr Nephrol. 2015;30(10):1807-13.

5 Riedel TJ, Knight J, Murray MS, Milliner DS, Holmes RP, Lowther WT. 4-Hydroxy-2-oxoglutarate aldolase inactivity in primary hyperoxaluria type 3 andglyoxylate reductase inhibition. Biochim Biophys Acta. 2012; 1822(10):1544-52.

6 Williams HE, Wandzilak TR. Oxalate synthesis, transport and the hyperoxaluric syndromes. JUrol. 1989;141(3 Pt 2):742-9.

7 Milliner DS, Harris PC, Lieske JC. Primary Hyperoxaluria Type 3. 2015 Sep 24. In: Adam MP, Ardinger HH, Pagon RA, et al., editors. GeneReviews ${ }^{\circledR}$ [Internet]. Seattle (WA): University of Washington, Seattle; 1993 2019. Available from: https://www.ncbi.nlm.nih.gov/books/NBK316514/

8 Wang X, Zhao X, Wang X, Yao J, Zhang F, Lang Y, et al. Two Novel HOGA1 Splicing Mutations Identified in a Chinese Patient with Primary Hyperoxaluria Type 3. Am J Nephrol. 2015;42(1):78-84.

9 Beck BB, Baasner A, Buescher A, Habbig S, Reintjes N, Kemper MJ, et al. Novel findings in patients with primary hyperoxaluria type III and implications for advanced molecular testing strategies. Eur J Hum Genet. 2013; 21(2):162-72. 
10 Monico CG, Rossetti S, Belostotsky R, Cogal AG, Herges RM, Seide BM, et al. Primary hyperoxaluria type III gene HOGA1 (formerly DHDPSL) as a possible risk factor for idiopathic calcium oxalate urolithiasis. Clin J Am Soc Nephrol. 2011;6(9):2289-95.

11 Williams EL, Bagg EA, Mueller M, Vandrovcova J, Aitman TJ, Rumsby G. Performance evaluation of Sanger sequencing for the diagnosis of primary hyperoxaluria and comparison with targeted next generation sequencing. Mol Genet Genomic Med. 2015;3(1):69-78.

12 Hoppe B. An update on primary hyperoxaluria. Nat Rev Nephrol. 2012;8(8):467-75.

13 Richard E, Blouin JM, Harambat J, Llanas B, Bouchet S, Acquaviva C, et al. Late diagnosis of primary hyperoxaluria type III. Ann Clin Biochem. 2017;54(3):406-11.

14 Tang X, Bergstralh EJ, Mehta RA, Vrtiska TJ, Milliner DS, Lieske JC. Nephrocalcinosis is a risk factor for kidney failure in primary hyperoxaluria. Kidney Int. 2015;87(3):623-31.

15 Cochat P, Rumsby G. Primary hyperoxaluria. N Engl J Med. 2013;369(7):649-58.

16 Clifford-Mobley O, Hewitt L, Rumsby G. Simultaneous analysis of urinary metabolites for preliminary identification of primary hyperoxaluria. Ann Clin Biochem. 2016;53(Pt 4):485-94.

17 Jacob DE, Grohe B, Gessner M, Beck BB, Hoppe B. Kidney stones in primary hyperoxaluria: new lessons learnt. PLoS One. 2013;8(8):e70617.

18 Ventzke A, Feldkotter M, Wei A, Becker J, Beck BB, Hoppe B. Systematic assessment of urinary hydroxy-oxoglutarate for diagnosis and follow-up of primary hyperoxaluria type III. Pediatr Nephrol. 2017;32(12): 2263-71. 